\title{
Evaluación de la Distribución Espacial de la Flora Silvestre de los Humedales de Ite, Provincia Jorge Basadre Grohmann 2008
}

RESPONSABLE: B/g. Luis Lloja Lozano

RESUMEN. Se evaluó la distribución espacial de la flora silvestre de la Zona de influencia (Pampa Baja) y el humedal de Ite, distrito de Ite, provincia de Jorge Basadre Grohmann y departamento de Tacna, realizada entre mayo del 2008 y febrero del 2009.

Para evaluar la distribución espacial de la flora silvestre utilizamos la metodología de los cuadrantes aleatorios de $1 x 1 \mathrm{~m}$, con un tamaño de muestra de 10 cuadrantes. En la evaluación seleccionamos especies claves: Grindelia glutinosa, Lippia nodiflora, Ambrosia peruviana, Cenchrus echinatus Cynodon dactylon, Bacopa monieri, Scirpus californicus, Thypha dominguensis, Paspalum vaginatum contabilizando un total de 9087 individuos.

En la evaluación se usaron parámetros como la media, varianza, indice de dispersión y nomograma de Clapham, llegando a concluir que la distribución es contagiosa en cada uno de las especies evaluadas. $\begin{aligned} \text { MIEMBros: } & \text { Blg. Víctor Carbajal Zegarra } \\ & \operatorname{lng} \text {. Hernán Hurtado Hurtado }\end{aligned}$

\begin{abstract}
The space distribution of the wild flora of the influence area was evaluated (Pampa Baja) and the humedal of Ite, district of Ite, country of Jorge Basadre Grohmann and department of Tacna, carried out among May from the 2008 to February of the 2009.
\end{abstract}

To evaluate the space distribution of the wild flora we used the methodology of the random quadrants of $1 x 1$ $m$, with a size of sample of 10 quadrants. In the evaluation we select key species: Glutinous grindelia, Lippia nodiflora, Ambrosia peruviana, Cenchrus echinatus Cynodon dactylon, Bacopa monieri, Scirpus californicus, Thypha dominguensis and Paspalum vaginatum counting a total of 9087 individuals.

In the evaluation it was used parameters like the Media, Variance, Dispersion index and Clapham nomograma; ending up concluding that the distribution is Contagious in each one of the evaluated species.
INTRODUCCIÓN. La definición de humedal más utilizada en la actualidad es la de la Convención de Ramsar: "extensiones de marismas, pantanos, tuberas o aguas de régimen natural o artificial, permanentes o temporales, estancadas o corrientes, dulces, salobres o saladas, incluyendo las extensiones de agua marina cuya profundidad en marea baja no excede de los seis metros" ( Ramsar, 1990). Además, la Convención ( artículo 2.1), agrega sobre la definición de los humedales: " podrán comprender sus zonas ribereñas o costeras adyacentes, así como islas o extensiones de agua marina de una profundidad superior a los seis metros en marea baja, cuando se encuentra dentro del humedal"

Los humedales de Ite, se encuentran a $90 \mathrm{~km}$ noroeste de la ciudad de Tacna en el distrito de Ite, Provincia de Jorge Basadre, de la región de Tacna; este ecosistema costero es una formación artificial producto de la deposición de materiales de relave minero sobre la playa, y de la ampliación de la frontera agrícola en el sector Ite Norte (Pulido \& Tabilo - Valdivieso, 2001 ). Este es último sistema lacustre costero significativo hasta el centro de Chile, $800 \mathrm{~km}$ al sur ( Scout \& Carbonell, 1986 ). Presenta una vegetación natural pantanosa, conformada en su mayoría por fanerógamas ( Zegarra, 1995 ). El proceso de formación data de 1960, desde entonces se convierte en refugio de aves acuáticas silvestres, tanto residentes como migratorias, que buscan descanso y alimentación en el lugar y además presenta vegetación silvestre.

El humedal de Ite constituye la zona de estudio más amplia, debido a la presencia de mayor diversidad de flora, lo que permite la existencia de fauna silvestre y domesticada (INRENA, 2000). Los humedales de Ite, son importantes debido a que sirven de estancia a las aves migratorias que se dirigen hacia el sur o hacia Norteamérica. Ite es el único humedal entre Mejía (Arequipa) y Huasco (Chile) (MORRIS y PANTY, 1999).

El origen del humedal se remonta al año 1940, cuando su ancho de playa no era mayor a $\operatorname{los} 80 \mathrm{~m}$ y contenía a una agricultura incipiente. Su formación se debe al estuario originado por el río Locumba, y así como también por los afloramientos del agua proveniente de las zonas agrícolas de Pampa Alta y Baja del distrito de Ite. Por los años 60, la SPCC comenzó el depósito de desechos mineros, provocando una alteración del ecosistema (MORRIS y PANTY, 1999).

En cuanto a la vegetación del humedal de Ite se considera que la estructura física está compuesta por plantas herbáceas y perennes, las cuales presentan patrones de distribución espacial que desconocemos, por lo que es necesario conocer la distribución espacial 
de la flora silvestre que predomina en el humedal de Ite. La distribución espacial permite tener conocimiento sobre la situación de las especies con respecto a la disponibilidad del hábitat y alimentación; en la naturaleza se encuentra tres tipos de distribución espacial: Distribución Uniforme, Distribución Aleatoria y Distribución Contagiosa (CANALES, 2004).

\section{MATERIAL Y MÉTODOS}

\section{Zona de Estudio}

La investigación fue realizado en el distrito de Ite, provincia de Jorge Basadre, de la región Tacna, tanto en el área de influencia (Pampa Baja) y el humedal. Dicha zona está ubicada en el valle de Ite a $115 \mathrm{~km}$. de Tacna, vía costanera. La cual está ubicada en el nivel ecológico bajo (Región ecográfica Costa o Chala de 0 a 80 m.s.n.m.). Este distrito se encuentra entre los $17^{\circ} 51^{\prime} 27^{\prime \prime}$ de latitud sur y $70^{\circ} 57^{\prime} 47^{\prime \prime}$ de longitud oeste.

\section{METODOLOGÍA}

Para la evaluación de la distribución espacial de las especies silvestres empleamos el método de los cuadrantes aleatorios. Dichos cuadrantes fueron determinados al azar, en un espacio de $10 \times 10 \mathrm{~m}$, haciendo un total de 10 puntos de muestreo, en donde posteriormente ubicamos un cuadrante más pequeño de $1 \times 1 \mathrm{~m}$ que mostró representatividad en cuanto a flora, con respecto al cuadrante superior. De estos cuadrantes pequeños, identificamos y contamos todas las especies allí existentes.

El muestreo se hizo en 05 etapas, cada dos meses, y se tuvieron 05 zonas de muestreo, donde en cada una de las estaciones de muestreo se hizo mediante el método de los cuadrantes aleatorios.

Las zonas de muestreo fueron Pampa Baja y los humedales de Ite. Las 2 primeras zonas de muestreo correspondieron al área de influencia Pampa Baja y las 3 últimas zonas de muestreo correspondieron al área de influencia de los humedales de Ite.

Las plantas han sido identificadas en el herbario Takana de la Facultad de Ciencias de la Universidad Nacional Jorge Basadre Grohmann de Tacna, de acuerdo con las descripciones de ANDERSON (2001), FERREIRA (1961), ZEGARRA (1992, 1994 y 1995), LEON, et al. (1995), y MEZA, et al. (1998) según a sus caracteres morfológicos.

Para la distribución espacial se usaron parámetros importantes como son: la frecuencia observada, media, varianza, índice de dispersión, grado de libertad, prueba de hipótesis, nomograma de Clapham.

\section{RESULTADOS}

Cuadro 1 Relación de especies de flora silvestre encontrados en Pampa Baja y los humedales, distrito de Ite, provincia de Jorge Basadre Grohmann, Tacna. 2008

\begin{tabular}{|l|l|c|c|}
\hline \multicolumn{1}{|c|}{ Especies } & \multicolumn{1}{|c|}{ N. común } & $\begin{array}{c}\text { Pampa } \\
\text { baja }\end{array}$ & Humedal \\
\hline Grindelia glutinosa & Chiñe & $X$ & \\
\hline Lippia nodiflora & Tiquil tiquil & $X$ & \\
\hline Ambrosia peruviana & Ambrosia & $X$ & \\
\hline Cenchrus echinatus & Cadillo & $X$ & \\
\hline Cynodon dactyion & Grama dulce & $X$ & \\
\hline Bacopa monieri & Verdolaga & & $X$ \\
\hline Scirpus californicus & Junco & & $X$ \\
\hline Thypha dominguensis & Totora & & $X$ \\
\hline Paspalum vaginatum & Grama salada & & $X$ \\
\hline & & & \\
\hline
\end{tabular}

Fuente: propia de los autores

Cuadro 2 Número de especies e individuos de la flora silvestre clave distribuidos en 10 cuadrante de $1 \mathrm{~m}^{2}$ en la zona de influencia (Pampa Baja), distrito de Ite, provincia de Jorge Basadre Grohmann, Tacna - 2008

\begin{tabular}{|l|l|l|l|l|l|l|l|l|l|l|l|l|}
\hline \multicolumn{1}{|c|}{ Especie } & $\begin{array}{c}\text { Nombre } \\
\text { comin }\end{array}$ & C1 & C2 & C3 & C4 & C5 & C6 & C7 & C8 & C9 & C10 & TOTAL \\
\hline Grindelia glutinosa & Chine & 0 & 0 & 8 & 3 & 0 & 4 & 0 & 0 & 5 & 8 & 28 \\
\hline Limpia nodiflora & Tiquil bquil & 3 & 0 & 7 & 4 & 0 & 5 & 0 & 0 & 0 & 8 & 27 \\
\hline Ambrosia penuviana & Ambrosia & 3 & 0 & 7 & 4 & 0 & 5 & 0 & 0 & 0 & 0 & 19 \\
\hline Cenchrus echinatus & Cadillo & 0 & 15 & 17 & 20 & 12 & 0 & 0 & 0 & 13 & 0 & 77 \\
\hline Cynodon dactyion & Grama duice & 0 & 4 & 0 & 0 & 9 & 513 & 176 & 112 & 22 & 16 & 852 \\
\hline TOTAL & 6 & 19 & 39 & 31 & 21 & 527 & 176 & 112 & 40 & 32 & 1003 \\
\hline
\end{tabular}

Fuente: propia de los autores

Cuadro 3 Número de especies e individuos de la flora silvestre clave distribuidos en 10 cuadrantes de $1 \mathrm{~m}^{2}$ en la zona del humedal de Ite, distrito de Ite, provincia de Jorge Basadre Grohmann, Tacna-2008

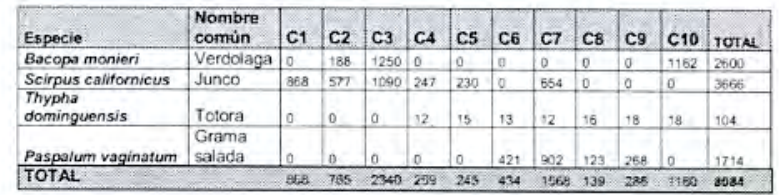

Fuente: propia de los autores

Cuadro 4 Análisis estadistico de la distribución espacial de Grindelia glutinosa en Pampa Baja, del distrito de Ite. provincia de Jorge Basadre Grohmann, Tacna - 2008

\begin{tabular}{|l|r|}
\hline Varianza & 9,960 \\
\hline Media & 2,800 \\
\hline Indice de Dispersión & 3,557 \\
\hline G.L. & 9,000 \\
\hline$X^{2}$ & 32,014 \\
\hline$X^{2} T a b$ & 16,900 \\
\hline
\end{tabular}

La distribución espacial encontrada es CONTAGIOSA, debido a que el indice de dispersión fue de 3.557 y la varianza es mayorque la media $(9,960$ y 2,800$)$. 
Cuadro 5 Análisis estadistico de la distribución espacial de Lippia nodiflora en Pampa Baja, del distrito de Ite, provincia de Jorge Basadre Grohmann, Tacna-2008

\begin{tabular}{|l|r|}
\hline Varianza & 9,010 \\
\hline Media & 2,700 \\
\hline Indice de Dispersión & 3,337 \\
\hline G.L. & 9,000 \\
\hline $\mathrm{X}^{2}$ & 30,033 \\
\hline $\mathrm{x}^{2} \mathrm{Tab}$ & 16,900 \\
\hline
\end{tabular}

La distribución espacial encontrada es CONTAGIOSA, debido a que el indice de dispersión fue de 3.337 y la varianza es mayor que la media $(9,010$ y 2,700$)$.

Cuadro 6: Análisis estadistico de la distribución espacial de Ambrosia peruviana en Pampa Baja, del distrito de Ite, provincia de Jorge Basadre Grohmann, Tacna - 2008

\begin{tabular}{|l|r|}
\hline Varianza & 12,040 \\
\hline Media & 1,600 \\
\hline Indice de Dispersión & 7,525 \\
\hline $\mathrm{G} . \mathrm{L}$. & 9,000 \\
\hline $\mathrm{x}^{2}$ & 67,725 \\
\hline $\mathrm{x}^{2} \mathrm{Tab}$ & 16,900 \\
\hline
\end{tabular}

La distribución espacial encontrada es CONTAGIOSA, debido a que el indice de dispersión fue de 7,525 y la varianza es mayor que la media $(12,040$ y 1,600$)$.

Cuadro 7. Análisis estadistico de la distribución espacial de Cenchrus echinatus en Pampa Baja, del distrito de Ite, provincia de Jorge Basadre Grohmann, Tacna - 2008.

\begin{tabular}{|l|r|}
\hline Varianza & 63,410 \\
\hline Media & 7,700 \\
\hline Indice de Dispersión & 8,235 \\
\hline G.L. & 9,000 \\
\hline $\mathrm{x}^{2}$ & 74,116 \\
\hline $\mathrm{x}^{2} \mathrm{Tab}$ & 16,900 \\
\hline
\end{tabular}

La distribución espacial encontrada es CONTAGIOSA, debido a que el índice de dispersión fue de 8,235 y la varianza es mayor que la media $(63,410$ y 7,700$)$.

Cuadro 8. Análisis estadístico de la distribución espacial de Cynodon dactylon en Pampa Baja, del distrito de Ite, provincia de Jorge Basadre Grohmann, Tacna - 2008

\begin{tabular}{|l|r|}
\hline Varianza & 23493,560 \\
\hline Media & 85,200 \\
\hline İndice de Dispersión & 275,746 \\
\hline G.L. & 9,000 \\
\hline $\mathrm{x}^{2}$ & 2481,714 \\
\hline $\mathrm{x}^{2} \mathrm{Tab}$ & 16,900 \\
\hline
\end{tabular}

La distribución espacial encontrada, es CONTAGIOSA, debido a que el índice de dispersión fue de 3.337 y la varianza es mayor que la media $(9,010$ y 2,700$)$.
Cuadro 9. Análisis estadístico de la distribución espacial de Bacopa monieri en humedales, del distrito de Ite, provincia de Jorge Basadre Grohmann, Tacna - 2008.

\begin{tabular}{|l|r|}
\hline Varianza & 227208,800 \\
\hline Media & 260,000 \\
\hline prdice de Dis ersión & 873,880 \\
\hline G.L. & 9,000 \\
\hline $\mathrm{x}^{2}$ & 7864,920 \\
\hline $\mathrm{x}^{2} \mathrm{Tab}$ & 16,900 \\
\hline
\end{tabular}

La distribución espacial encontrada es CONTAGIOSA, debido a que el indice de dispersión fue de 873,880 y la varianza es mayor que la media $(227208,800$ y 260,000).

Cuadro 10: Análisis estadístico de la distribución espacial dè Scirpus californicus en humedales, del distrito de Ite, provincia de Jorge Basadre Grohmann, Tacna - 2008.

\begin{tabular}{|l|r|}
\hline Varianza & 147212,240 \\
\hline Media & 366,600 \\
\hline Índice de Dispersión & 401,561 \\
\hline G.L. & 9,000 \\
\hline $\mathrm{x}^{2}$ & 3614,048 \\
\hline $\mathrm{x}^{2} \mathrm{Tab}$ & 16,900 \\
\hline
\end{tabular}

La distribución espacial encontrada es CONTAGIOSA, debido a que el indice de dispersión fue de 401,561 y la varianza es mayor que la media $(147212,240$ y 366,600$)$.

Cuadro 11: Análisis estadistico de la distribución espacial de Thypha dominguensis en humedales, del distrito de Ite,provincia de Jorge Basadre Grohmann, Tacna - 2008

\begin{tabular}{|l|r|}
\hline Varianza & 50,440 \\
\hline Media & 10,400 \\
\hline Índice de Dispersión & 4,850 \\
\hline G.L. & 9,000 \\
\hline $\mathrm{x}^{2}$ & 43,650 \\
\hline $\mathrm{x}^{2} \mathrm{Tab}$ & 16,900 \\
\hline
\end{tabular}

La distribución espacial encontrada es CONTAGIOSA, debido a que el indice de dispersión fue de 4,850 y la varianza es mayor que la media $(50,440$ y 10,400$)$.

Cuadro 12: Análisis estadistico de la distribución espacial de Paspalum vaginatum en humedales, del distrito de Ite, provincia de Jorge Basadre Grohmann, Tacna - 2008

\begin{tabular}{|l|r|}
\hline Varianza & 78401,840 \\
\hline Media & 171,400 \\
\hline Índice de Dispersión & 457,420 \\
\hline G.L. & 9,000 \\
\hline $\mathrm{x}^{2}$ & 4116,783 \\
\hline $\mathrm{x}^{2} \mathrm{Tab}$ & 16,900 \\
\hline
\end{tabular}


La distribución espacial encontrada es CONTAGIOSA debido a que el indice de dispersión fue de 457.420 y la varianza es mayor que la media $(78401,840$ y 171,400)

\section{DISCUSIÓN}

Si bien los tipos de vegetación que se repiten en distintas zonas y situaciones son en cierto modo similares, no existen dos espacios ocupados por comunidades idénticas. Esto se debe, en parte al hecho de que la composición florística varía continuamente. Es decir, es casi imposible determinar objetivamente los límites entre las distintas expresiones de la vegetación, puesto que sus elementos no son discretos, como lo son, por ejemplo, los organismos. En algunas situaciones, es posible trazar un límite entre dos tipos de vegetación, cuando el cambio súbito de algún factor o grupo de factores ambientales determina un cambio brusco de la vegetación. En este caso se habla de discontinuidad espacial amontonado (MATEUCCI y COLMA, 1982).

La flora de Pampa Baja y los humedales de Ite, responden $\mathrm{y}$ ha respondido a los cambios en las condiciones imperantes en ellos. Prueba de ello ha sido la modificación en la composición florística, documentada, por ejemplo, a través de la ausencia de ciertas especies. Posibles explicaciones para estas desapariciones incluyen la reducción del tamaño de los humedales como así también de Pampa Baja durante los últimos años, la extracción selectiva de algunas especies con valor económico y la alteración del ambiente por contaminación y otros tipos de impacto humano. También es importante reconocer que las fluctuaciones en el nivel y calidad de agua son naturales en todos los humedales. Tales fluctuaciones contribuyen a los cambios florísticos y del paisaje, en los que algunas especies pueden eliminarse mientras que otras aumentan en abundancia (LEÓN, et al. 1995).

En el Cuadro 9, respecto a Bacopa monieri, la distribución espacial encontrada es CONTAGIOSA, debido a que el índice de dispersión fue de 873,880 y la varianza es mayor que la media $(227208,800$ y 260,000). Esta especie es típica de lugares húmedos encontrando condiciones más favorables a orillas del humedal. Comparando los resultados con al estudio de ORIHUELA et al. (2004), hay una variación en cuanto a la cantidad de individuos.

Como observamos en el Cuadro 1, las zonas de Pampa Baja y el humedal presentan 5 y 4 especies clave respectivamente. ORIHUELA, et al. (2004) no evaluaron a Lippia nodiflora, Grindelia glutinosa, Cenchrus echinatus y Cynodon dactylon; como especies-clave. Siendo consideradas Sesuvium portulacastrum, Lippia nodiflora, Ambrosia peruviana y Trifolium repens como las especies clave de esos ecosistemas.

En el Cuadro 2, se observa el número de especies (5) e individuos (1003) encontrados en diez cuadrantes evaluados en la zona de Pampa Baja, siendo el Cynodon dactylon la especie que presentó la mayor densidad poblacional (852) y la especie de menor densidad poblacional fue la especie Ambrosia peruviana (19) en comparación con los individuos evaluados (815) por ORIHUELA, et al. (2004) utilizando la misma metodología.

La mayor presencia de la especie Cynodon dactylon nos indica que existen factores aceptables de hábitat para su desarrollo (CANALES, 2004), asi mismo parece que los nutrientes que necesita esta especie provienen de terrenos agrícolas cercanos, ORIHUELA, et al. (2004).

En el Cuadro 3, se observa el número total de especies (4) e individuos (8084) en diez cuadrantes evaluados en la zona del humedal Ite. Asi mismo se puede observar la densidad poblacional de Scirpus californicus con 3666 individuos, seguida por Bacopa monieri con 2600 individuos, siendo similares los resultados con el trabajo de ORIHUELA, et al. (2004) que evaluaron un total de 55805 individuos de la especie Bacopa monieri. Esta disminución puede deberse a que esta especie ocupa rápidamente micrositios temporalmente soleados, pero es reemplazada por otras especies que prosperan a su sombra, LEÓN, et al. (1995). Con respecto a Thypha dominguensis es vegetación característica de sitios inundados como los humedales (LEÓN, et al.1995).

En el Cuadro 4, la distribución espacial encontrada es CONTAGIOSA, debido a que el índice de dispersión fue de $3.557 \mathrm{y}$ la varianza es mayor que la media $(9,960 \mathrm{y}$ 2,800). La presencia de Grindelia glutinosa, en la zona de Pampa Baja, se debe a que esta especie es capaz de desarrollarse en condiciones de estrés hídrico, que es una caracteristica de esta zona, teniendo como fuente de agua algunos escurrajes de cultivos aledaños (ZEGARRA, 1999). Cabe mencionar que en documentos anteriores no se ha considerado a esta especie en la misma zona (ORIHUELA, et al. 2004). SANCHEZ, et al. (1999) registran a Grindelia como un género que también se distribuye en la zona altoandina. En el Cuadro 5, la distribución espacial encontrada es CONTAGIOSA, debido a que el índice de dispersión fue de $3.557 \mathrm{y}$ la varianza es mayor que la media $(9,010 \mathrm{y}$ 2,700). La presencia de Lippia nodiflora en zona de Pampa Baja se debe a que esta especie es más frecuente en los bordes de acequias o terrenos de cultivo MORRIS y PANTY, 1999. Haciendo una comparación con los resultados obtenidos en el 2004 podemos encontrar que el promedio de esta especie ha disminuido (X 2004, $=119,10$ ), esto se debería a actividades agrícolas, pues es considerada como maleza (MINISTERIO DE AGRICULTURA, 1999).

En el Cuadro 6, la distribución espacial encontrada es CONTAGIOSA, debido a que el índice de dispersión fue de 7,525 y la varianza es mayor que la media (12,040 y 1,600). Ambrosia peruviana, la podemos encontrar compitiendo con plantas cultivadas en esta localidad debido a su mayor necesidad de agua. Haciendo una comparación con los resultados obtenidos por ORIHUELA, et al. (2004), podemos encontrar que el promedio de esta especie ha disminuido (X 2004= 
$81,50)$, esto puede deberse a actividades agrícolas ya que también es considerado como maleza (MINISTERIO DEAGRICULTURA, 1999).

En el Cuadro 7, la distribución espacial encontrada es CONTAGIOSA, debido a que el índice de dispersión fue de 8,235 y la varianza es mayor que la media $(63,410$ y 7,700). El cadillo Cenchrus echinatus, es una especie que necesita de humedad constante, por ello podemos encontrarla en terrenos cultivados y aledaños a estos PERUAGRO, 2004. Además esta planta posee semillas de alto poder germinativo.

La distribución espacial encontrada, es CONTAGIOSA, debido a que el índice de dispersión fue de 275,746 y la varianza es mayor que la media (23493,560 y 85,200). La grama dulce (Cynodon dactylon), es una especie que podemos encontrarla en terrenos cultivados y aledaños a estos, esta especie podemos encontrarla asociada con cultivos de alfalfa compitiendo por el agua.

En el Cuadro 9, respecto a Bacopa monieri, la distribución espacial encontrada es CONTAGIOSA, debido a que el índice de dispersión fue de 873,880 y la varianza es mayor que la media $(227208,800$ y 260,000). Esta especie es típica de lugares húmedos encontrando condiciones más favorables a orillas del humedal. Comparando los resultados con al estudio de ORIHUELA et al. (2004), hay una variación en cuanto a la cantidad de individuos.

En el Cuadro 10, la distribución espacial encontrada es CONTAGIOSA, debido a que el índice de dispersión fue de 401,561 y la varianza es mayor que la media (147212,240 y 366,600). La especie Scirpus californicus es típica de humedales. A comparación del documento de ORIHUELA, et al. (2004) podemos observar una disminución de individuos, esto posiblemente se debe a algún desplazamiento provocado por el pastoreo o por el incremento de la abundancia de totora.

En el Cuadro 11, la distribución espacial encontrada es CONTAGIOSA, debido a que el índice de dispersión fue de 4,850 y la varianza es mayor que la media $(50,440 \mathrm{y}$ 10,400), Gran parte de la especie Thypha dominguensis fue sembrado por SPCC con el objetivo de mitigar los efectos causados por los relaves (BALVIN, 1992). El tipo de distribuciones contagiosa o aglomerada, lo que nos indica que la especie está amenazada en cuanto a disponibilidad y calidad de hábitat. (KREBS, 1986). Quizás la totora sea la especie con mayor potencial de uso que pueda haber en la zona, pues según (LOZA, 2000) es utilizado para la construcción de balsas, artesanias, alimento, forraje, sustrato de reproducción de peces, hábitat de especies de aves migratorias y residentes, que algunas veces se alimentan de su semilla y raíces en la producción de abono orgánico, purifica aguas contaminadas y protege de la erosión del suelo.

En el Cuadro 12, la distribución espacial encontrada en Paspalum vaginatum es CONTAGIOSA, debido a que el índice de dispersión fue de 457,420 y la varianza es mayor que la media $(78401,840$ y 171,400$)$. Es una especie que ocupa varios ecosistemas gracias a su rapido crecimiento y tendencia a trepar sobre otras plantas (LEÓN, etal. 1995).

\section{CONCLUSIÓN}

La distribución espacial de las especies: Grindelia glutinosa, Lippia nodiflora,Ambrosia peruviana, Cenchrus echinatus Cynodon dactylon, Bacopa monieri,Scirpus californicus, Thypha dominguensis, Paspalum vaginatum es de tipo contagiosa.

\section{REFERENCIAS BIBLIOGRÁFICAS}

BALVIN, D., TEJEDO, J., CARDENAS, F. 1992. "Contaminación por metales pesados tóxicos producidos por las operaciones minero - metalúrgicas de Southern Perú Copper Corporation". X Congreso Nacional de Biología (2 - 7 de Agosto de 1992).

BLANCO, D. E. 1999. "Los humedales como hábitat de aves acuáticas”. pp. 208-217. En: Malvárez, A. I. (Ed.). Tópicos sobre humedales subtropicales y templados de Sudamérica. ORCYT-UNESCO, Montevideo.

BRACK, A. y MENDIOLA, C. 2000. Ecologia del Perú, Asociación Editorial Bruño, Lima, 495 pp.

CANAlES G., A. 2004. Ecología. Teoría - Práctica. Ediciones Gráfica, Puno. 273 pp.

FERREIRA, R. 1990. "Fitogeografia del Perú". Boletín Sociedad Geográfica de Lima. 164 (6): 119-124.

HELMERS, D. 1992. Shorebird. Management Manual. WHSRN. Manomet.

KREBS, CH. J. 1986. Ecología. Análisis experimental de la distribución y abundancia. Segunda edición. Edit. Pirámide. S.A, Madrid, $782 \mathrm{pp}$

LAVALLEE D., JULIEN, M. y POZZI-ESCOT, 2004. Proyecto "PERU SUR". Ministerio de Relaciones Exteriores-Francia CNRS-Francia.

LEON, B.; YOUNG, K. y A. CANO. 1995. La Flora Vascular de los Pantanos de Villa. Lima, adiciones y guía para las especies comunes. Publicación Museo Historia Natural UNMSM (B) 38:1-39.

LOZA DEL CARPIO, 2003. "Guía Metodología de Educación Ambiental de la Reserva Nacional del Titicaca". Ministerio de Agricultura - Instituto Nacional de Recursos Naturales.

MESA, A.; MUÑOZ, M. y R. PINTO. 1998. Presencia de Nolana andansonii (Roemer y Schultes) Johnst. y Nolana intonsa Johnst Historia Natural, Chile (333):3-7.

MINISTERIO DE AGRICULTURA, INSTITUTO NACIONAL DE RECURSOS NATURALES, INRENA, 1996. "Estrategia Nacional para la conservación de humedales en el Perú". 
MORRIS M. Y PANTY O. 1999. Espacio y conciencia geográfica en Tacna. Ed. Tèrcer Milenio .

NIERING, W. 1985. Wetlands. The Audubon Society Nature Guides. Alfred A. Knopf, Inc. New Cork.

ORIHUELA, Y., NINA, M., BARBACHAN, A., ESQUIVEL, W., ESQUIVEL, S., VERGARA, J. y TICONA, A. 2004. Distribución Espacial de la Flora Silvestre en el Área de Influencia de los Humedales de Ite.

PERUAGRO, 2004. Publicación Nacional Agraria \& Agronegocios. Impresión Alpigraf.

RAMSAR. 1990. Procceding of the Third Meeting of the Conference of the Contracting Parties. Ramsar Convention Bureau, UICN, Gland, Suiza.

\section{ANEXOS}

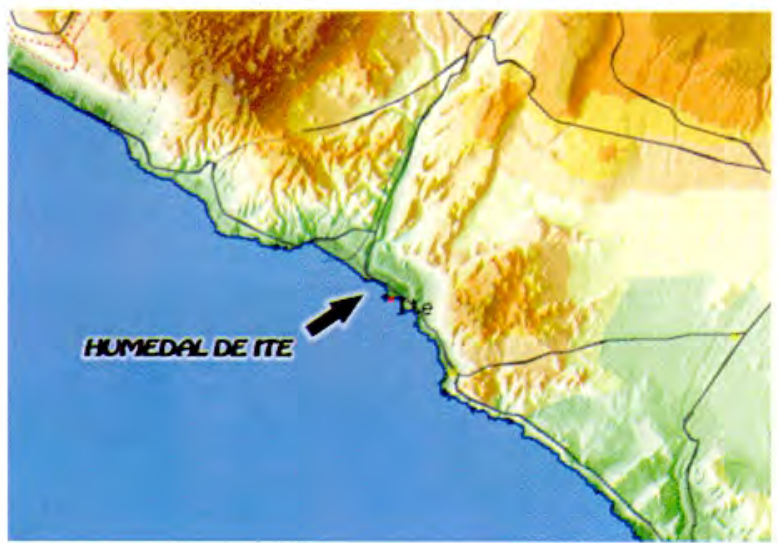

Figura $\mathrm{N}^{\circ} 1$ : Localización geográfica del área en estudio, zona de influencia (Pampa Baja) y humedal de Ite. (Falling Rain Genomics, Inc. 1996-2004)

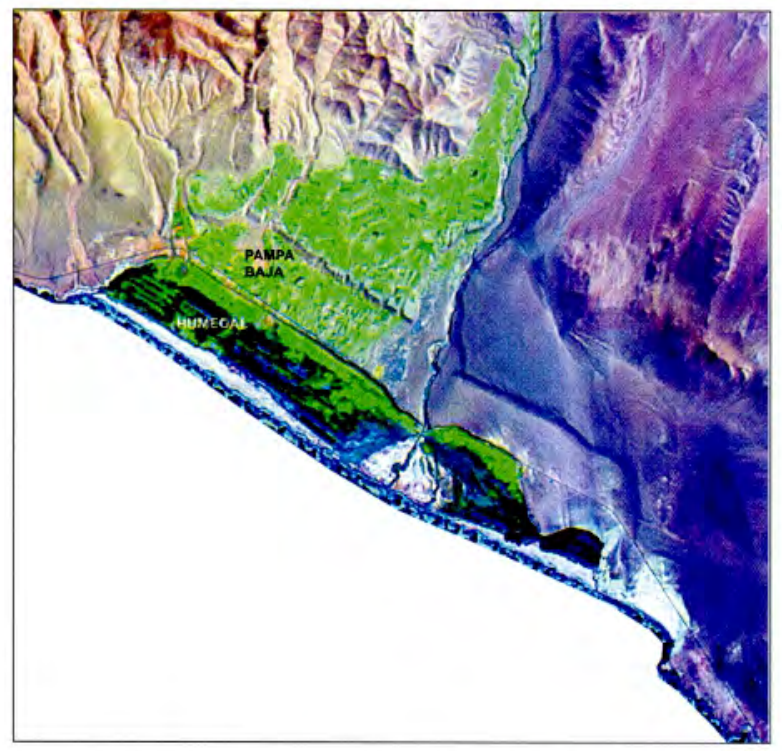

Figura $\mathrm{N}^{\circ}$ 02. Localización geográfica del área en estudio, zona de influencia (Pampa Baja) y humedal de Ite. (Falling Rain Genomics, Inc. 1996-2004)
SÁNCHEZ, F., CAPCHA, A., CRUZ, J., SÁNCHEZ, J., DURAN, A. y QUISPE, U.1999. Evaluación de Impacto Ambiental del Humedal "La Moya" Ayaviri-Puno.

SCOTT, D. \& M. CARBONELL (Comps.). 1986. Inventario de humedales de la Región Neotropical. IWRB Slimbridge and UICN Cambridge.

TABILO-VALDIVIESO, E. 2003. El Beneficio de los Humedales en la Región Neotropical. Centro Neotropical de Entrenamiento en Humedales. La Serena, Chile.

ZEGARRA, 1999 ZEGARRA, R. 1995. "La Vegetación Pantanosa de Ite". Revista Ciencia y Desarrollo. UNJBG (1):12:15.

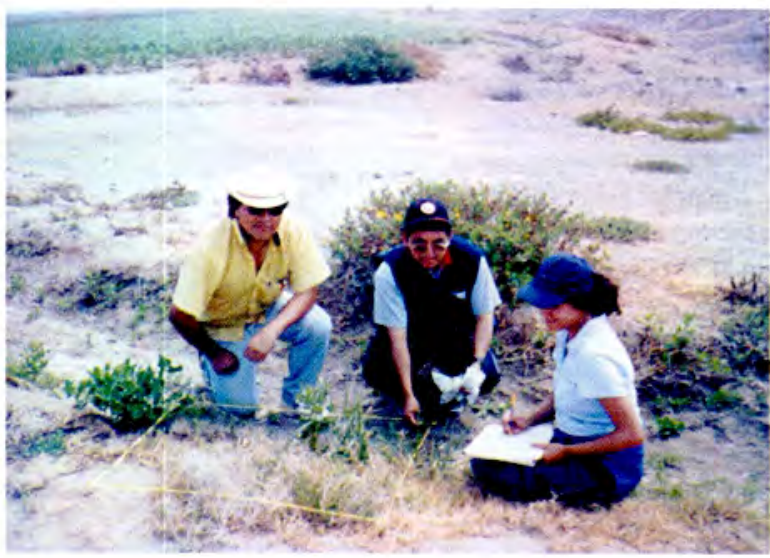

Figura $\mathrm{N}^{\circ}$ 3: Delimitación del cuadrante $(1 \times 1 \mathrm{~m})$. Para la determinación de la distribución espacial de la flora.

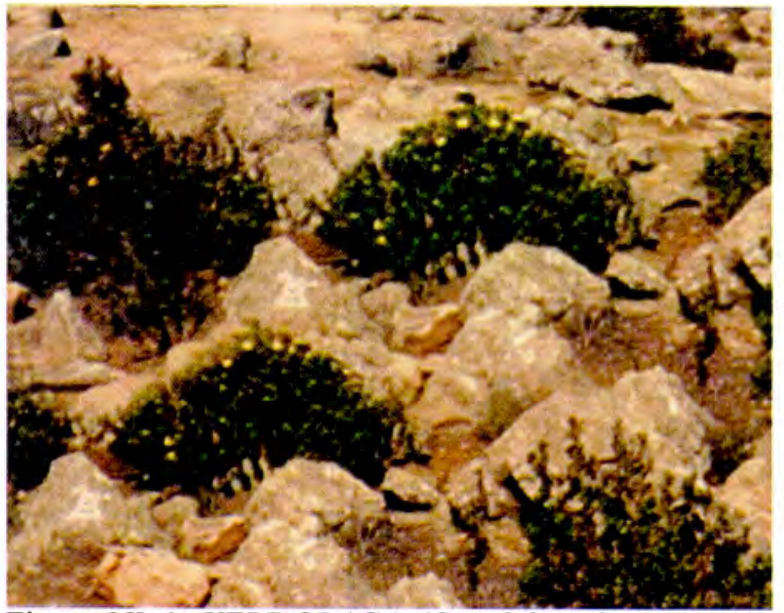

Figura $\mathrm{N}^{\circ}$ 4: VERDOLAGA (Grindelia glutinosa). Se observa una distribución espacial contagiosa. 